\title{
IS NEUTROPHILS / LYMPHOCYTES RATIO AFFECTED DURING 'MIGRAINE ATTACKS WITH AURA' IN MIGRAINE PATIENTS?
}

\author{
Mehlika Panpalli Ates ${ }^{1}$
}

\author{
${ }^{1}$ Department of Neurology, University of Health Sciences, Diskapi Yildirim Beyazit Training and Research Hospital, \\ Ankara, Turkey
}

Address for Correspondence: Mehlika Panpalli Ates, E-mail: muefhulkika@gmail.com Received: 20.02.2021; Accepted: 30.03.2021; Available Online Date: 27.05.2021

(C) Copyright 2021 by Dokuz Eylül University, Institute of Health Sciences - Available online at https://dergipark.org.tr/en/pub/jbachs

Cite this article as: Panpalli Ates M. Is Neutrophils/Lymphocytes Ratio Affected During 'Migraine Attacks with Aura' in Migraine Patients?J Basic Clin Health Sci 2021; 2: 36-40.

\begin{abstract}
Purpose: The formation and pathophysiology of migraine attacks with aura are still ambiguous. Sterile inflammation is claimed to play a role in the pathogenesis of migraine. Neutrophil / lymphocyte ratio (NLR) is an inexpensive and practical indicator that can be well detectable from a complete blood count (CBC). This study, it was aimed to compare NLR in migraine patients, during attacks with aura, and without attack. Methods: A total of 98 patients diagnosed with migraine headache with aura and 34 healthy individuals without migraine were analyzed. All subjects were evaluated in terms of age, gender, CBC. NLR results were compared with migraine attacks with aura, periods without attacks, and 34 healthy controls.

Results: The 98 patients, migraine with aura, had higher NLR values during attacks than migraine-free episodes and controls ( $p=0.000, p=0.000$, respectively).

Conclusions: NRL is a marker of inflammation, and we determined that NLR increases in migraine attacks especially as in other acute inflammatory situations. Pain can be prevented by taking, non-steroidal antiinflammatory drugs (NSAIDs) and triptans which inhibit neurogenic inflammation and neuroinflammation in the cascade, early at the onset of attacks.
\end{abstract}

Keywords: Headache, inflammation, inflammatory markers, migraine, Neutrophil / Lymphocyte Ratio

\section{INTRODUCTION}

Among the pains that affect people, headache is the most common. And the headache is the most common neurological condition in the world. In headache groups, migraine prevalence in the 15-55 age group was $16.4 \%$ [1].

Numerous mechanisms are thought to be liable for migraine pathogenesis.

Migraine is a primary neuronal process, triggered neuronal depolarization, and consequently spreading cortical depression wave (CSD) to be the underlying mechanism of the migraine aura [2].
Neuro-inflammatory situations, cytokines, various neuropeptides, and vasomotor changes are reported to be liable for migraine pathogenesis. Reduced antioxidant levels in migraine patients are thought to increase oxidative stress, thereby causing inflammation and changes, causing pathophysiological effects in the vessels [3]. In addition, during migraine attacks, inflammation occurs in some brain tissues arising from neuronal activation and following secretion of proinflammatory neuropeptides from perivascular nerve terminals $[3,4]$. During repeated migraine attacks, perivascular inflammation can make a possible increased risk of 
Table 1. Demographic and clinical characteristics of patients

\begin{tabular}{|c|c|c|c|}
\hline & $\begin{array}{c}\text { Migraine Group } \\
(98)\end{array}$ & $\begin{array}{c}\text { Control Group } \\
\text { (34) }\end{array}$ & $p$ \\
\hline Age (years) & $28.9 \pm 7.22$ & $28.6 \pm 6.47$ & \\
\hline (mean \pm SD) & $(18-41)$ & $(18-41)$ & 0.875 \\
\hline Gender (Female) & $100 \%$ & $100 \%$ & \\
\hline Duration of Migraine (year) & $\begin{array}{c}4.1 \pm 1.93 \\
(1-7)\end{array}$ & - & \\
\hline Average Attack Frequency (last 3 months) & $4.3 \pm 4.2$ & - & \\
\hline$\geq 4$ Attacks per month & $51(58.2 \%)$ & - & \\
\hline$<4$ Attacks per month & $41(41.8 \%)$ & - & \\
\hline
\end{tabular}

stroke in migraine $[3,5]$. When the migraine attacks begin, trigeminal nerve stimulation and blood flow increases, protein leakage from the vessels, and vasoactive peptides are composed as a result of neurogenic inflammation. Therefore, sterile inflammation plays a role in the pathogenesis of migraine $[3,6,7]$.

The response of leukocytes to the stress is an increase in neutrophil and a decrease in lymphocyte counts. Therefore, the neutrophil / lymphocyte ratio (NLR), which has been used recently, is used as markers of systemic inflammation and oxidative stress $[8,9]$.

NLR is an inexpensive and practical indicator that can be well detectable from a CBC [8]. As well as, NLR as a subclinical inflammatory indication is related to mortality and the prognosis of numerous diseases $[8,10-12]$. It has been shown that NLR has prognostic value, especially high NLR levels are associated with poor prognosis $[8,13]$.

In previous studies, patients with migraine and the control groups, or among migraine patients, (those with episodic migraine attacks and with chronic pain patients) were compared [3,6,14] each other. This study is different from the others. In this study, personal differences were eliminated by comparing the results of the same episodic migraine patients. It was analyzed in the two-period; when there was migraine attack with an aura, and interictal migraine attack periods. In addition, the attack and interictal periods of the same migraine patients' results were compared with the healthy control group without headache.

This study, it was aimed to evaluate NLR changes, known as an important inflammatory marker, and compare them during migraine attacks with aura and interictal periods of the same patients to healthy controls without migraine.

\section{METHODS}

We retrospectively recruited 98 patients with migraine with aura according to ICHD III-beta criteria who were followed in the neurology outpatient clinic [15]. 34 healthy volunteers without migraines or other types of headache were included in the study as a control group.

Migraine patients were evaluated for headache diaries, face-to-face interviews, and file records. Only female episodic migraine patients with a disease duration of 1-7 years, with an attack frequency below 15 , and only with visual aura were included in the study.

In the study, migraine diagnosed 98 patients', during migraine attacks with aura and non-attack periods, examination results were reviewed, and CBC results, especially NLR results were compared between groups.

The migraine frequency increases in women two to four times compared to men. Only women with migraines were included in the study to eliminate gender difference because migraine attacks are more common in women and it is also stated that female 
sex hormones have a role in migraine pathophysiology [16].

Patients who have been exposed to blood transfusions or other blood products, have uncontrolled hypertension, intracranial mass lesions, have been treated for anemia or polycythemia in the past year, and have had a history of bleeding or surgery for the past seven months, hematological, chronic kidney and liver disorders, pregnancy or fever history and oncological diseases in the last month, iron deficiency anemia, thalassemia, metabolic and inflammatory bowel disorders, diabetes mellitus, thyroid disease, chronic alcohol use, vitamin B12 and folic acid deficiency/uses were not included in the study. The control group was also composed of people without primary and secondary headaches, with similar exclusion criteria.

The study is retrospective; it was carried out according to the Helsinki Declaration.

\section{Statistical Analysis}

Statistical analysis was realized using an Statistical Package for Social Sciences (SPSS®) 22.0 for Windows and Mac os $\mathrm{x}$. Shapiro-Wilk test was used to test the hypothesis of normality. Numeric data were presented as mean \pm standard deviation and nominal data were presented as percentage (\%). Student ttest for paired and unpaired data was used for the statistical analysis within and between the groups in the basal assessment of the blink reflex. Pearson's correlation test and Spearman rank correlation coefficient were used respectively for parametric and non-parametric data to assess any correlations between the neurophysiological and clinical parameters. $p$ values less than 0.05 were accepted as statistically significant.

\section{RESULTS}

The blood samples (CBC, white blood cell count (WBC), neutrophil, lymphocyte, hemoglobin, and platelet values) of 98 patients who applied to the neurology outpatient clinic with a migraine attack with aura were recorded. The blood samples of the same patients were compared with the painless period within 3 months. In addition, the results of the same patients' were compared with 34 healthy control groups without headaches. Migraine patients and the control group included in the study were all females. 98 female patients diagnosed migraine with aura [mean age $28.9 \pm 7.22$ years (18-41)] and 34 healthy

Table 2. Comparison of laboratory test results between groups

\begin{tabular}{|c|c|c|c|c|c|c|}
\hline & $\begin{array}{l}\text { Migraine } \\
\text { Attack with }\end{array}$ & $\begin{array}{l}\text { Migraine } \\
\text { Interictal }\end{array}$ & $\begin{array}{l}\text { Control Group } \\
\text { (Group 3) }\end{array}$ & & $p$ & \\
\hline & $\begin{array}{c}\text { Aura } \\
\text { (Group 1) }\end{array}$ & $\begin{array}{l}\text { Period } \\
\text { (Group 2) }\end{array}$ & & $\begin{array}{c}\text { Group 1- } \\
2\end{array}$ & $\begin{array}{c}\text { Group 1- } \\
3\end{array}$ & $\begin{array}{c}\text { Group } 2- \\
3\end{array}$ \\
\hline $\begin{array}{c}\text { NLR } \\
(\text { mean } \pm \text { SD) }\end{array}$ & $2.9 \pm 1.16$ & $1.8 \pm 0.63$ & $1.9 \pm 0.39$ & 0.000 & 0.000 & 0.173 \\
\hline $\begin{array}{c}\text { WBC } \\
(\text { mean } \pm \text { SD) }\end{array}$ & $8.8 \pm 2.14$ & $7.2 \pm 1.59$ & $7.1 \pm 1.17$ & 0.000 & 0.000 & 0.913 \\
\hline $\begin{array}{l}\text { Neutrophil } \\
\text { (mean } \pm \text { SD) }\end{array}$ & $5.8 \pm 1.84$ & $4.1 \pm 1.13$ & $4.2 \pm 0.81$ & 0.000 & 0.000 & 0.635 \\
\hline $\begin{array}{l}\text { Lymphocyte } \\
\text { (mean } \pm \text { SD) }\end{array}$ & $2.1 \pm 0.55$ & $2.3 \pm 0.55$ & $2.2 \pm 0.43$ & 0.013 & 0.601 & 0.351 \\
\hline
\end{tabular}


volunteers [mean age $28.6 \pm 6.47$ years (18-41)] were included in the study ( $p=0.875)$.

The average attack frequency of migraine patients in the last 3 months was $4.6 \pm 4.3$. While the frequency of attacks was $\geq 4$ / month in 51 patients $(58.2 \%)$, the attack frequency was $<4 /$ month in 41 patients $(41.8 \%)$ (Table 1).

In the migraine attack with aura mean NLR $(2.9 \pm$ $1.16)$ is higher than the interictal period $(1.8 \pm 0.63)$ and the control group $(1.9 \pm 0.39)(p=0.000, p=$ 0.000 , respectively). But the mean NLR in the migraine group during the interictal period was not statistically different from the control group $(p=0.173)$ (Table 2).

Mean WBC value compared between groups; in migraine attack with aura $(8.8 \pm 2.14)$, it was found to be higher than the interictal period $(7.2 \pm 1.59)$ and the control group $(7.1 \pm 1.17)(p=0.000, p=0.000$, respectively). The mean WBC value in the migraine interictal period was not statistically different from the control group ( $p=0.913$ ) (Table 2).

The mean neutrophil value was higher in the migraine attack with aura $(5.8 \pm 1.84)$ than in the interictal period $(4.1 \pm 1.13)$ and the control group $(4.2 \pm 0.81)$ ( $p=0.000, p=0.000$; respectively). The mean neutrophil value in the migraine interictal period was not statistically different from the control group $(p=$ 0.635) (Table 2).

The mean lymphocyte value was smaller in a migraine attack with aura $(2.1 \pm 0.55)$ than in the interictal period $(2.3 \pm 0.55)(p=0.013)$. The mean lymphocyte value during the attack with migraine aura and in the interictal period did not differ statistically from the control group $(2.2 \pm 0.43)(p=0.601, p=$ 0.351 ; respectively) (Table 2 ).

When the correlation between migraine attack period with aura and age, NLR, WBC, neutrophil, lymphocyte, was examined; It was found to be a slight correlation between NLR and neutrophil value $(p=$ 0.021, Rho: 0.216 and $p=0.03$, Rho: 0.202 ; respectively).

When the correlations between NLR and the values determined in the patient group were examined; there was a correlation between WBC ( $p=0.000$, Rho: 0.525), neutrophil $(p=0.000$, Rho: 0.765$)$, lymphocyte $(p=0.000$, Rho: -0.564$)$.

\section{DISCUSSION}

In this study, serum NLR was increased in migraine attacks with aura compared with interictal migraine patients and matched control subjects.
The findings of this study support the putative role of inflammation in migraine.

Presently, the being of neurogenic inflammation has been demonstrated in migraine models, and triptans, effective therapeutic drugs, are used to prevent neurogenic inflammation [17].

It has been stated that migraine comes about as a result of the neurogenic inflammatory reaction and during migraine attacks, some biomarkers increased as a signal of inflammation [18].

Findings suggesting migraine pain is a type of sterile inflammation have been reported [19].

Serum NLR levels are commonly used biomarkers of peripheral inflammation and oxidative stress in chronic neurological diseases. Neuroinflammation is related to the pathogenesis of some neurodegenerative neurological disorders like Parkinson's and Alzheimer's disease. [10,11]. As well, high NLR values have been reported to be an independent indicator of prognosis in cardiovascular diseases, cerebrovascular, and cancer [8,11-13].

The migraine aura arises as a result of cortical spreading depression. The course that triggers off cortical spreading depression not entirely clear. The release of inflammatory molecules by emotional stress is thought to play a role here [19].

In the study, the CBC results during the visual aura of the migraine group for the painful attack were examined. During the migraine attack with aura, it was observed that there were lower serum lymphocyte levels and higher serum NLR levels compared to the interictal period.

In this study, by removing personal differences, we found that NLR changes in migraine patients were affected, particularly throughout migraine attacks with aura.

In addition, we found that NLR was higher in patients with migraine attacks compared to controls. These results may indicate that inflammation increases in migraines, especially during an aura attack. As NSAIDs and triptans inhibit neurogenic inflammation and neuroinflammation in the cascade, pain can be prevented by taking them early at the onset of the attack.

Conflict of Interest: None

Ethical approval: The research is retrospective study. The study was carried out according to the 1964 Helsinki Declaration.

Informed Consent: For the study, written and verbal consent was obtained from the patients.

Financial Disclosure: None

Peer-review: Externally peer-reviewed. 


\section{REFERENCES}

1. Yücesan C. Migraine and Treatment. J NeurolSpecial Topics. 2008;1(1):10-21

2. Dodick DW. A Phase-by-Phase Review of Migraine Pathophysiology. Headache. 2018;58:416.

3. Vanmolkot F, de Hoon J. Increased C-Reactive Protein in Young Adult Patients with Migraine. Cephalalgia. 2007;27(7):843-846.

4. Waeber C, Moskowitz MA. Migraine as an inflammatory disorder. Neurology 2005;64:9-15.

5. Welch KM. Stroke and migraine-the spectrum of cause and effect. Funct Neurol 2003;18:121-126.

6. Muhammed J, Halim SA, Hitam W, Tharakan J. Migraine with aura complicated by "migraine triggered seizures" and "occipital lobe infarction": A case report. Neurology Asia 2014;19(3):323326

7. Ramachandran R. Neurogenic inflammation and its role in migraine. Semin Immunopathol. 2018;40(3):301-314.

8. Ates U, Bahadir K, Ergun E, et al. Determination of Pentraxin 3 levels in diagnosis of Appendicitis in children. Pediatr Int. 2020;62(5):624-8.

9. Azab B, Bhatt VR, Phookan J, et al. Usefulness of the neutrophil-to-lymphocyte ratio in predicting short- and long-term mortality in breast cancer patients. Ann Surg Oncol. 2012;19(1):217-24.

10. Huang WJ, Zhang X, Chen WW. Role of oxidative stress in Alzheimer disease. Biomed Rep. 2016;4(5):519-22.

11. Yazar T, Yazar HO. Evalution of C-reactive protein/albumin ratio according to stage in patients with idiopathic Parkinson disease. Turk J Neurol. 2019;25(3):123-8.

12. Tekin S, Avcı E, Nar R, et al. Are Monocyte/HDL, Lymphocyte/Monocyte and Neutrophil/Lymphocyte Ratios Prognostic or Follow-up Markers in Ischemic Cerebrovascular Patients?. J Basic Clin Health Sci 2020;4(1):038043.

13. Belotti A, Doni E, Bolis S, et al. Peripheral blood lymphocyte/monocyte ratio predicts outcome in follicular lymphoma and in diffuse large B-cell lymphoma patients in the rituximab era. Clin Lymphoma Myeloma Leuk. 2015;15(4):208-213.

14. Karabulut KU, Egercioglu TU, Uyar M, Ucar Y. The change of neutrophils/lymphocytes ratio in migraine attacks: A case-controlled study. Ann Med Surg (Lond). 2016;10:52-56.
15. Headache Classification Committee of the International Headache Society (IHS). The International Classification of Headache Disorders, 3rd edition (beta version). Cephalalgia. 2013;33:629-808.

16. Vetvik KG, MacGregor EA. Sex differences in the epidemiology, clinical features, and pathophysiology of migraine. Lancet Neurol. 2017;16(1):76-87.

17. Buzzi MG, Moskowitz MA. The antimigraine drug, sumatriptan (GR43175) selectively blocks neurogenic plasma extravasation from blood vessels in dura mater $\mathrm{Br} J$ Pharmacol. 1990;99(1):202-206.

18. Moskowitz MA, Cutrer FM. Sumatriptan: a receptor-targeted for migraine, Annu. Rev. Med. 1993;44:145-154.

19. Burstein R, Noseda R, Borsook D. Migraine: multiple processes, complex pathophysiology. J Neurosci. 2015;35(17):6619-6629. 